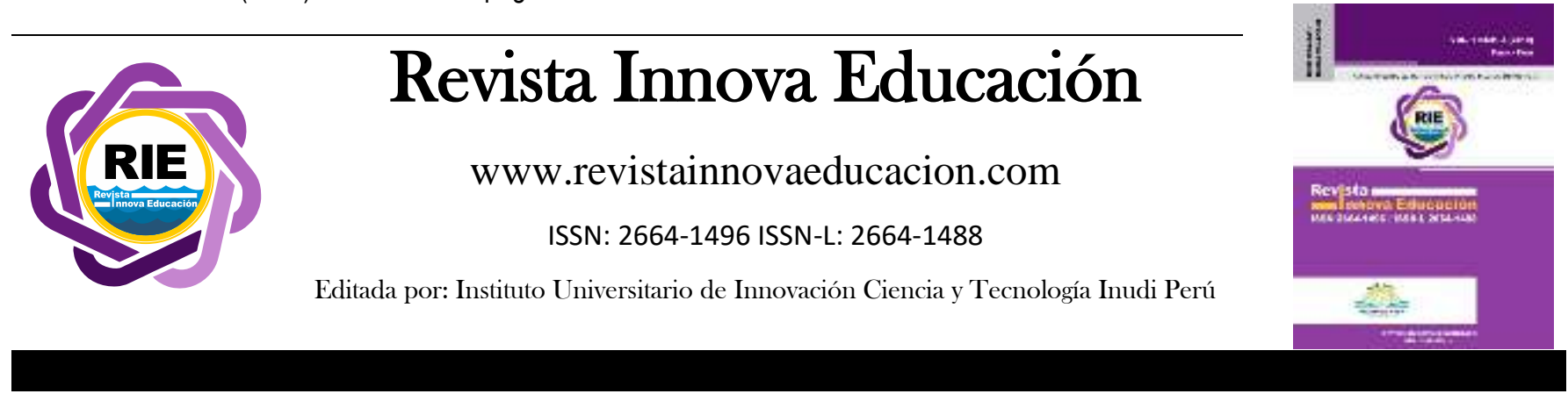

\title{
Déficit de atención y comprensión lectora de textos andinos en niños que estudian a moderada altitud
}

\author{
Attention deficit and reading comprehension of Andean texts in children who study at moderate \\ altitude
}

\section{Grecia Mamani ${ }^{1}$}

Universidad Nacional del Altiplano de Puno, Puno-Puno, Perú

(D) https://orcid.org/0000-0001-5817-4896

\author{
Ana Vilca \\ Universidad Nacional del Altiplano de Puno, Puno-Puno, Perú \\ (D) https://orcid.org/0000-0003-4809-7334 \\ Fred Torres \\ Universidad Nacional del Altiplano de Puno, Puno-Puno, Perú \\ Dhttps://orcid.org/0000-0003-0834-6834
}

DOI: https://doi.org/10.35622/j.rie.2020.04.006

Recibido 16/08/2020/ Aceptado 30/09/2020 Publicado 21/10/2020

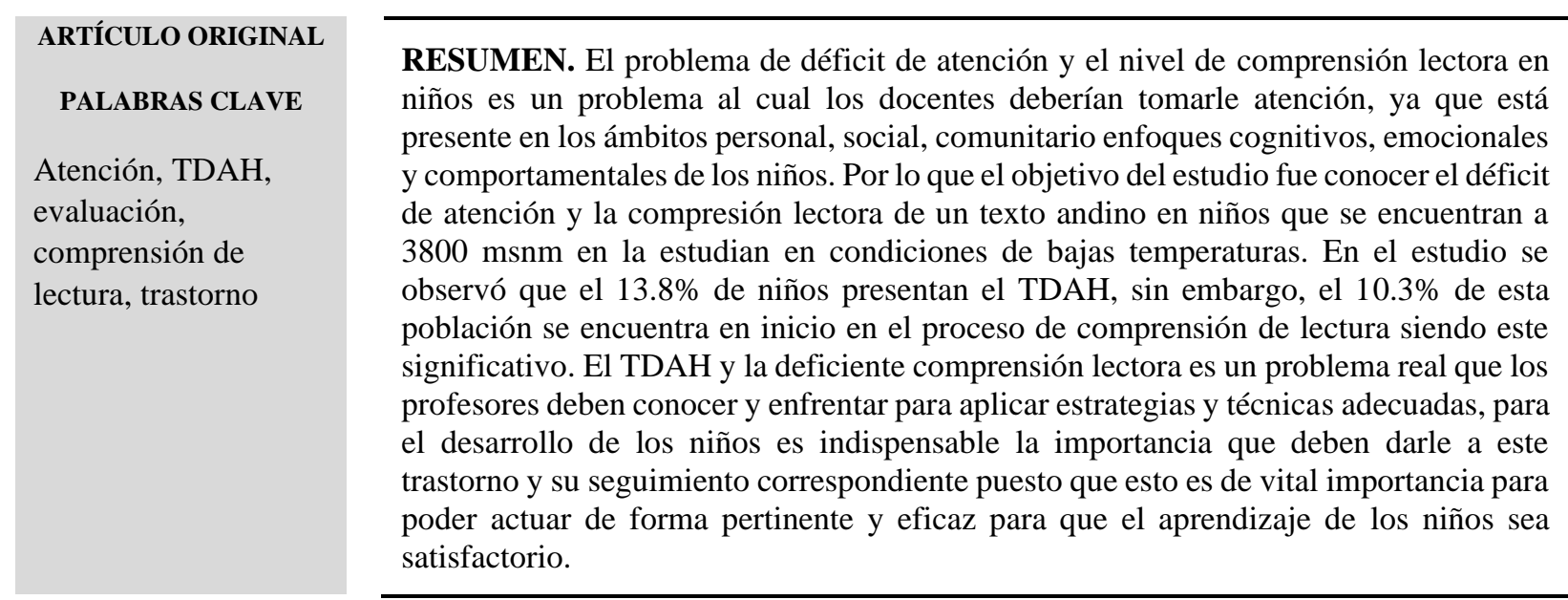

KEYWORDS

${ }^{1}$ Correspondencia: greciavianeymv@gmail.com
ABSTRACT. The problem of attention deficit and the level of reading comprehension in children is a problem that teachers must pay attention to; since it is present in the 
Attention, ADHD, assessment, reading comprehension, disorder personal, social and community areas and cognitive, emotional and interactive behaviors of children. Therefore, the objective of the study was to know the attention deficit and the reading comprehension of an Andean text in children who are at 3800 meters above sea level when they study it in conditions of low temperatures. In the study, it was observed that $13.8 \%$ of children have ADHD; however, $10.3 \%$ of this population is at the beginning of the reading comprehension process, this being significant. ADHD and meager reading comprehension is a real problem that teachers must know and face in order to apply appropriate strategies and techniques, for the development of children it is essential the importance that they must give to this disorder and its corresponding monitoring since this is of vitally important to be able to act in a relevant and effective way so that children's learning.

\section{INTRODUCCIÓN}

El déficit de atención y la comprensión lectora de textos andinos en niños son dificultades de aprendiza frecuentes en los salones de clases. La falta de atención pareciera inminente porque a primera vista se aprecia la inatención, falta de concentración, la impulsividad y la hiperactividad que se presentan en los niños y niñas a la hora de leer un texto contextualizado. Lo que representaba un factor que no estaba permitiendo una comprensión de lectura satisfactoria o significativa, por lo que decidimos realizar este estudio que se enfoca en el déficit de atención y la comprensión lectora la cual se encuentra descrita en el Currículo Nacional de Educación Básica del Perú, de acuerdo a las capacidades y desempeños que establece el Ministerio de Educación del Perú como norma vigente en la elaboración del presente trabajo.

Una de las más grandes problemáticas educativas en el Perú es que los estudiantes desde temprana edad no tienen una buena comprensión lectora y se hace evidente en las múltiples pruebas como son: $\mathrm{PISA}^{2}, \mathrm{ECE}^{3}$ en las que no se alcanza los parámetros mínimos que indiquen y reflejen una buena comprensión lectora. El Perú se encuentra entre los antepenúltimos lugares en la comprensión lectora de los estudiantes tanto de educación primaria como de secundaria de acuerdo a lo observado en el informe peruano de resultados en PISA ${ }^{1}$. Desde los lineamientos del Currículo Nacional se pretende mejorar, pero aún falta las condiciones de adecuación contextualizada de los estándares de aprendizaje propuestos por el Ministerio de Educación del Perú, concernientes a la comprensión de lectura en el área de comunicación. Es muy probable que el TDAH ${ }^{4}$ sea un factor que esté afectando a la comprensión lectora de los niños y niñas. El CNEB ${ }^{5}$ en el área de comunicación establece las capacidades para evaluar la comprensión lectora, 1) Obtiene información del texto escrito, 2) Infiere e interpreta información del texto, 3) Reflexiona y evalúa la forma contenido y contexto del texto (Ministerio de Educación, 2016).

Según American Psychiatric Association (2012) el trastorno de déficit de atención es un desorden neurológico que según cifras oficiales de la OMS afectan entre el 5\% y 7\% de los niños en todo el mundo. La idea principal que plantea Ferrer et al. (2017) en referencia al TDAH nos indica que es de índole observacional generalmente por los docentes y los familiares del estudiante. $\mathrm{Y}$ al respecto de las características de este trastorno Torres et al. (2016) nos dice que las

\footnotetext{
${ }^{2}$ PISA: por sus siglas en ingles Programme for International Student Assessment (prueba de programa para la evaluación internacional de estudiantes)

${ }^{3}$ ECE: evaluación censal de estudiantes.

${ }^{4}$ TDAH: trastorno de déficit de atención

${ }^{5}$ CNEB: Currículo Nacional de la educación Básica.
} 
implicaciones como en lo cognitivo, emocional, comportamental y en ámbitos personal, social, comunitario y escolar, siendo este último un foco de interés sobresaliente por las dificultades significativas que se presentan a este nivel. Y por otra parte Christien et al.(2013) realiza una conclusión diciendo que siendo este uno de los problemas de salud más relevantes de cuantos afectan al funcionamiento de los niños y adolescentes es el Trastorno por Déficit de Atención e Hiperactividad TDAH.

El TDAH es un trastorno que tiene una etiopatogenia heterogénea es decir que puede ser causada por una variedad de condiciones biológicas, psicológicas y sociales las cuales pueden actuar en conjunto o individualmente pero serán estudios futuros los que aporten más conocimiento acerca de cómo interactúan estas causas variadas entre sí y el papel de los factores ambientales en su conjunto (Barra \& García 2005 citado por Alza, 2013).

El TDAH es un trastorno neurobiológico que aqueja tanto a adultos como a niños, es descrito como un patrón persistente o continuo de inatención y/o hiperactividad e impulsividad que impide las actividades diarias o el desarrollo típico, los individuos con TDAH también pueden experimentar dificultades para mantener la atención, en la memoria de trabajo, la función ejecutiva (habilidad del cerebro para comenzar una actividad, organizarse y llevar a cabo tareas), el TDAH se sitúa en la categoría de Trastornos de inicio en la infancia, la niñez o la adolescencia (Centro Nacional de Recursos de CHADD 2013). Como también la FundacionCADAH.org (2012) consolida que el trastorno de déficit de atención es un desorden neurológico que según cifras oficiales de la OMS afectan entre el 5\% y 7\% de los niños en todo el mundo. Y refiriéndonos a una información nacional la asociación APDA (2002) nos dice que es una condición neuropsiquiatría caracterizada por la presencia de déficit de atención, hiperactividad-impulsividad o de ambos. El TDAH se caracteriza por la presencia de una triada sintomática caracterizada por hiperactividad, impulsividad e inatención. Los síntomas se presentan de modo heterogéneo en uno $\mathrm{u}$ otro paciente, con mayor o menor intensidad para uno u otro componente de la triada (Centro Nacional de Recursos de CHADD, 2013).

De acuerdo con la Fundacion CADAH (2012) el déficit de atención que se presenta más en la edad escolar en el nivel de educación primaria el cual, no permite un aprendizaje adecuado y significativo. Afirmando esta condición a este problema toma relevancia porque dificulta el aprendizaje del niño o niña, la conducta intensa y curiosa de los niños con alta capacidad exacerbada por el aburrimiento en clase (Barra \& García 2005 citado por Alza, 2013). Los niños hiperactivos, como tienen un estilo cognitivo impulsivo aplican menor número y variedad de estrategias de solución de problemas; Los niños resuelven los rompecabezas cogiendo al azar piezas y funcionando por ensayo-error de una forma poco sistemática (no prueban primero con una pieza todas las posibilidades ni la apartan para continuar el mismo proceso con otra), o resuelven los problemas con los amigos siguiendo una única estrategia (pegar, amenazar o escaparse), aunque ésta no resulte eficaz (Orjales, 1999).

Según Garcia, M et al (2008) nos dice que la falta de atención y la escasa capacidad de controlar su actividad motora provocan una disminución del rendimiento escolar que conduce en la mayoría de los casos al fracaso escolar. Así mismo se debe tomar en cuenta que es crucial adaptar las exigencias escolares a las características y limitaciones de estos niños, que hasta en un 
$50 \%$ de los casos presentan también retraso motor y a veces un retraso del lenguaje (Martínez, 2016). Por lo que señalamos que este problema está presente y no está permitiendo un aprendizaje significativo entonces es importante saber cómo se presenta, para que maestros y maestras puedan tomar metodologías y estrategias didácticas para contrarrestar las comorbilidades que pueden presentarse de añadirá con el trastorno TDAH.

Es necesario que el docente de aula deba conocer el TDAH para dar respuestas a las necesidades de estos niños, el conocimiento por parte del profesorado del TDAH les ayuda a observar y recoger información de las conductas del alumno, a planificar y aplicar la intervención educativa adecuada para estos niños y es importante que se ponga en marcha un programa de aprendizaje. Según Espina \& Ortego (2004) afirma que asistir a aula de apoyo con personal terapéutico, profesor o pedagogo terapéutico $\left(\mathrm{PT}^{6}\right)$ es necesario en la mayoría de los casos de TDAH.

El TDAH tiene sus presentaciones clínicas según el DSM $-5^{7}$ Patrón persistente de inatención y/o hiperactividad-impulsividad que interfiere con el funcionamiento o desarrollo que se caracteriza por (1) y/o (2) que los caracteriza.

\section{Presentación clínica de falta de atención}

- No presta atención a los detalles o comete errores por descuido.

- Tiene dificultad para mantener la atención.

- Parece no escuchar.

- Tiene dificultad para seguir las instrucciones hasta el final.

- Tiene dificultad con la organización.

- Evita o le disgustan las tareas que requieren un esfuerzo mental sostenido.

- Pierde las cosas.

- Se distrae con facilidad.

- Es olvidadizo para las tareas diarias (Centro Nacional de Recursos de CHADD, 2013).

\section{Presentación clínica hiperactivo/impulsivo}

- Mueve o retuerce nerviosamente las manos o los pies, o no se puede quedar quieto en una silla.

- Tiene dificultad para permanecer sentado.

- Corre o se trepa de manera excesiva; agitación extrema en los adultos.

- Dificultad para realizar actividades tranquilamente.

- Actúa como si estuviera motorizado; el adulto frecuentemente se siente impulsado por un motor interno.

- Habla en exceso.

- Responde antes de que se haya terminado de formular las preguntas.

- Dificultad para esperar o tomar turnos.

- Interrumpe o importuna a los demás (Centro Nacional de Recursos de CHADD, 2013).

\section{Presentación clínica inatento e hiperactivo-impulsivo combinado}

\footnotetext{
${ }^{6}$ PT: Personal terapéutico.

${ }^{7}$ DSM-5: Manual Diagnóstico y Estadístico de los Trastornos Mentales quinta edición
} 
- El individuo presenta síntomas de ambas presentaciones clínicas mencionadas (Centro Nacional de Recursos de CHADD, 2013).

Lo que podemos resumir en la tabla 1.

Tabla 1 : Presentaciones de Déficit de Atención

\begin{tabular}{clll}
\hline \multicolumn{1}{c}{ TDAH } & \multicolumn{1}{c}{ PRESENTACIONES } & \multicolumn{2}{c}{ CATEGORÍAS } \\
\cline { 2 - 4 } & Falta de atención & Nada & 0 \\
\hline \multirow{2}{*}{$\begin{array}{c}\text { Trastorno de déficit de } \\
\text { atención }\end{array}$} & Hiperactividad/ impulsividad & Poco & 1 \\
\cline { 2 - 3 } & $\begin{array}{l}\text { Falta de atención e } \\
\text { hiperactividad /impulsividad }\end{array}$ & Bastante & 3 \\
\cline { 2 - 3 } & &
\end{tabular}

Fuente: Centro Nacional de Recursos de CHADD (2013)

DSM (Diagnostic and Statistical Manual of Mental Disorders) es el Manual Diagnóstico y Estadístico de los Trastornos Mentales quinta edición de la Asociación Americana de Psiquiatría (American Psychiatric Association, APA).

Según Torres (2011) la comprensión supone la construcción de una representación del significado global en la que el estudiante pone en juego distintos procesos, operaciones y habilidades, realizar síntesis, inferencias e interpretaciones para que le permita darle significados personales a la lectura. La comprensión lectora no solo se logra con el simple hecho de que el estudiante pueda decodificar con precisión sino más bien una tarea de gran complejidad en la que están implicados diferentes procesos cognitivos desde la percepción visual de signos gráficos hasta la construcción de una representación semántica de su significado en suma es la habilidad que posee el lector para extraer información de un texto impreso (Aliaga, 2012). Por eso es importante ver la relación del déficit de atención en el contexto de la comprensión lectora.

Según Vallés (2005) para comprender textos escritos se ponen en funcionamiento una serie de procesos de índole perceptiva (informativo de acceso a la lectura, en la lectura y su comprensión intervienen procesos perceptivos. El aprendizaje de la lectoescritura es el área en el que más se observa estas dificultades: escritura en espejo, mala grafía, inadecuada organización del espacio. La dislexia es el doble de frecuente en el TDAH lo cual aumenta las probabilidades de fracaso escolarmente (Espina \& Ortego, 2004).

En esta perspectiva, la memoria a largo plazo y las rutinas de acceso a la información cobran un papel muy relevante, y determinan el éxito o grado de logro que pueda tener el lector (Vallés, 2005).

La Competencia lee diversos tipos de textos escritos implica la combinación de las siguientes capacidades que están definidas por el currículo nacional como son a) Obtiene información del texto escrito, b) Infiere e interpreta información del texto, c) Reflexiona y evalúa, el contenido y contexto del texto escrito (Ministerio de Educación, 2016).

Lo que se puede resumir en la tabla 2 y 3. 
Tabla 2. Capacidades y Desempeños de Lectura en Lengua Materna

\begin{tabular}{|c|c|c|}
\hline COMPETENCIA & CAPACIDADES & DESEMPEÑOS \\
\hline \multirow{3}{*}{$\begin{array}{c}\text { Comprende textos } \\
\text { narrativos }\end{array}$} & $\begin{array}{l}\text { Obtiene información } \\
\text { del texto escrito }\end{array}$ & $\begin{array}{l}\text { Obtiene información } \\
\text { ubicada en distintas } \\
\text { partes del texto de } \\
\text { estructura simple, temas } \\
\text { del ámbito local, } \\
\text { acompañados con } \\
\text { algunas ilustraciones y } \\
\text { con vocabulario } \\
\text { conocido y variado. }\end{array}$ \\
\hline & $\begin{array}{l}\text { Infiere e interpreta } \\
\text { información del t exto } \\
\text { escrito }\end{array}$ & $\begin{array}{l}\text { Infiere relacionando } \\
\text { información explicita e } \\
\text { implícita e interpreta el } \\
\text { tema y el propósito del } \\
\text { texto, las intenciones del } \\
\text { autor. }\end{array}$ \\
\hline & $\begin{array}{l}\text { Reflexiona y evalúa la } \\
\text { forma, el contenido y } \\
\text { contexto del texto. }\end{array}$ & $\begin{array}{l}\text { Suponen que el } \\
\text { estudiante compara y } \\
\text { contrasta } \\
\text { formales y de contenido } \\
\text { del texto con la } \\
\text { experiencia, } \\
\text { Asimismo, emite una } \\
\text { opinión personal sobre } \\
\text { aspectos formales, } \\
\text { estéticos, contenidos de } \\
\text { los textos }\end{array}$ \\
\hline
\end{tabular}

Fuente: Ministerio de Educación (2016).

Tabla 2. Valoración de la compresión lectora

\begin{tabular}{lll}
\hline EVALUACIÓN & EVALUACIÓN & VALORACIÓN \\
NUMÉRICA & NOMINAL & LITERAL \\
\hline $18-20$ & AD & Logro destacado \\
$14-17$ & A & Logro esperado \\
$11-13$ & B & En proceso \\
$0-10$ & $\mathrm{C}$ & En inicio \\
\hline
\end{tabular}

Fuente: Ministerio de Educación (2016). 
Las valoraciones de competencias mediante la escala de evaluación al ser descriptivas son el resultado de un juicio docente realizado basado en el desempeño demostrado por el estudiante, en las diversas situaciones significativas planteadas por el docente. Dichas evaluaciones deben explicar el progreso del estudiante en un período determinado con respecto al nivel esperado de la competencia (estándares de aprendizaje) señalando avances, dificultades y recomendaciones para superarlos. En ese sentido no son notas aisladas ni promedios ni frases sueltas ni un adjetivo calificativo (Ministerio de Educación, 2016).

\section{MÉTODO Y MATERIALES}

El estudio que se realizó fue de tipo cuantitativo de carácter descriptivo - no experimental. En el estudio se tuvo una población de 133 niños y niñas de la Institución Educativa Pública "Sagrado Corazón de Jesús" que se encuentra ubicado geográficamente por sobre los 3800 msnm, teniendo temperaturas por debajo de los $6^{\circ}$ centígrados lo que representa una población única en la que se realizó este estudio. La muestra fue seleccionada por conveniencia seleccionando a un $26.31 \%$ de la población siendo esta representativa de la población. Los niños y niñas que asisten a esta institución provienen de los diferentes distritos de la localidad, con una condición socioeconómica media-baja, según las referencias de las nóminas de matrícula de la institución.

Para el proceso de recolección de datos se solicitó la autorización correspondiente a la dirección de esta institución. Luego se procedió a informar a los docentes y padres de familia de la sección seleccionada sobre los objetivos del estudio en el que estos aceptaron la participación de sus hijos y se procedió a firmar el consentimiento informado. Antes de aplicar los instrumentos previstos para el estudio, los niños y niñas fueron informados sin embargo 06 niños y niñas no lograron participar en el estudio por que presentaban enfermedades respiratorias agudas, dada la estación de invierno en la ciudad de puno teniendo como sujetos de estudio a 18 niñas y 11 niños.

Para la medición del déficit de atención se utilizó la guía de observación desarrollado por Manual Diagnóstico y Estadístico de los Trastornos Mental (DSM 5) de la Asociación Americana de Psiquiatría. quienes propone la medición con la escala de puntuación con tres criterios falta de atención, hiperactividad/Impulsividad e inatención e hiperactividad/impulsividad combinado, utilizando puntuaciones entre (0 - 3) desde nada, poco, bastante y mucho (Manual Conners, 2013). Y para evaluar la compresión de lectura se tomó el libro de comprensión lectora Cuentos, Mitos y Relatos, pensamiento vigente de la Cultura Andina elaborado por (Rivas, 2009) . la prueba escrita constó de 10 preguntas se clasificaron en categorías como son en nivel literal, inferencial y critico reflexivo las cuales posteriormente fueron clasificadas las respuestas obtenidas de los estudiantes en inicio lo que nos indica a los profesores que el estudiante muestra un progreso mínimo en una competencia y en este caso la comprensión lectora, evidencia con frecuencia dificultades en el desarrollo de las tareas, por lo que necesita mayor tiempo de acompañamiento, en proceso cuando el estudiante esta próximo o cerca al nivel esperado respecto a la comprensión de textos narrativos, para lo cual requiere acompañamiento y comprensión esperada (Ministerio de Educación, 2016).

Las escalas de evaluación para el diagnóstico del trastorno por déficit de atención e hiperactividad, así como para la distinción de los subtipos diseñadas sobre la base de los criterios del Manual Diagnóstico y Estadístico de Trastornos Mentales (quinta edición) son aceptados en todo el mundo por sus propiedades psicométricas sólidas. Los cuestionarios favorecen el registro 
de aspectos conductuales y constituyen una forma de evaluación del comportamiento La calificación se realiza con una escala tipo Likert, donde se asigna una puntuación a cada reactivo de 0 a 3. A mayor puntuación, más frecuentes serán los síntomas observados (Manual Conners, 2013). Como afirma Herrán et al. (2014) El cuestionario ampliamente utilizado son los de Conners. Entonces existe una confiable garantía de la aplicación del instrumento. Los criterios para el diagnóstico de los subtipo combinado según el DSM5 se tienen que tomar en cuenta seis o más indicadores de déficit de atención y al mismo tiempo seis o más indicadores de hiperactividad e impulsividad (Balbuena, 2014). Según Manual Conners, (2013) concluye sobre la escala de conners, en la que una puntuación de 17 o superior para los niños y de 13 o superior para las niñas hace sospechar la existencia de una posible TDAH. Para el análisis de datos se utilizó estadígrafos descriptivos de frecuencias, ponderación, porcentajes, media aritmética, desviación estándar. Para todos los cálculos se adoptó una significancia del 95\% en todo el proceso de análisis estadístico. El análisis se realizó con el SPSS v.21.0.

\section{RESULTADOS}

Se presenta los resultados de las variables es fruto de la observación de la variable, déficit de atención y la aplicación de una prueba para la segunda variable en los niños. Entendiéndose que para evidenciar los resultados los valores porcentuales de la población de estudio en la Tabla 4 que nos muestra la prevalencia del TDAH en un $13.8 \%$ y con respecto al Nivel Inicial de Compresión Lectora en la población es de $69 \%$, por lo que podemos explicar que el $10.3 \%$ de la población de estudio presenta un déficit de atención y al mismo tiempo déficit de compresión lectora de textos andinos.

Tabla 3. TDAH y Comprensión lectora en niños y niñas

\begin{tabular}{llrrrrrr} 
& & \multicolumn{4}{c}{ TDAH } & \multicolumn{2}{c}{ Total } \\
\cline { 3 - 8 } & & SIN TDAH & \multicolumn{2}{c}{ TDAH } & \multicolumn{1}{c}{$\boldsymbol{i} \%$} \\
& Inicio $<=\mathbf{1 0 , 0 0}$ & 17 & $58.6 \%$ & 3 & $10.3 \%$ & 20 & $69.0 \%$ \\
TDAH * & Proceso 11,00 - 13,00 & 6 & $20.7 \%$ & 0 & $0.0 \%$ & 6 & $20.7 \%$ \\
NCL & Esperado 14,00 - 17,00 & 2 & $6.9 \%$ & 1 & $3.4 \%$ & 3 & $10.3 \%$ \\
& Destacado 18,00+ & 0 & $0.0 \%$ & 0 & $0.0 \%$ & 0 & $0.0 \%$ \\
& Total & 25 & $86.2 \%$ & 4 & $13.8 \%$ & 29 & $100.0 \%$ \\
\hline
\end{tabular}

Leyenda: TDAH: Trastorno de Déficit de Atención e Hiperactividad; NCL: Nivel de Comprensión Lectora, Clasificación al aplicar la prueba de comprensión lectora y la guía de observación

Fuente: Elaboración propia

Este $10.3 \%$ de niños y niñas representa una población significativa que presenta este trastorno al que se deberá prestar atención para poder mitigar este problema en los salones de clases, para evaluar de manera objetiva y tener en cuenta otras variables al momento de atacar estas presentaciones del TDAH, observamos que en la Tabla 5 la presencia del TDAH según el sexo. La atención que deberán tener a los niños deberá ser preponderante por la frecuencia en la que se presenta este trastorno.

Tabla 4. TDAH por sexo en niños y niñas del altiplano

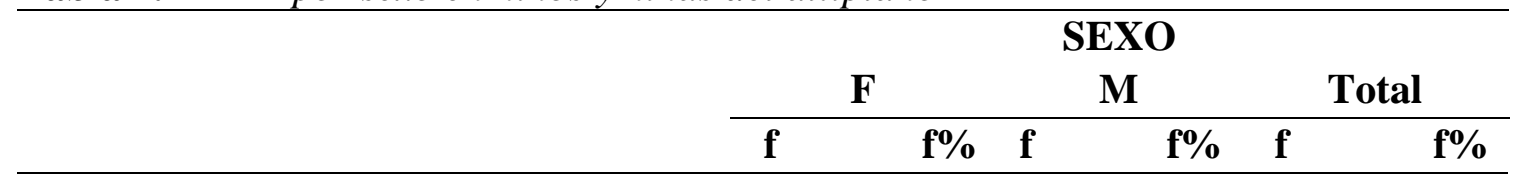




\begin{tabular}{llcrcccc}
\multirow{2}{*}{ TDAH } & SIN TDAH & 17 & $58.6 \%$ & 8 & $27.6 \%$ & 25 & $86.2 \%$ \\
& TDAH & 1 & $3.4 \%$ & 3 & $10.3 \%$ & 4 & $13.8 \%$ \\
\hline
\end{tabular}

Leyenda: TDAH: Trastorno de Déficit de Atención e Hiperactividad

Fuente: Elaboración propia

La presentación del TDAH en los sujetos de estudio pueden explicarse mejor según los indicadores analizados (Tabla 6) la falta de atención es el indicador más elevado ya que existe un $20 \%$ de esta condición dividida en un $17.2 \%$ en niñas y un $3.4 \%$ en niños, siendo este indicador al que debemos de tomar importancia por ser el más elevado, sin embargo en el cuadro combinado de inatención e hiperactividad/Impulsividad también se puede observar una frecuencia considerable a tomar en cuenta para atacar estos puntos para contrarrestar el TDAH.

Tabla 6. Indicadores del TDAH según Sexo

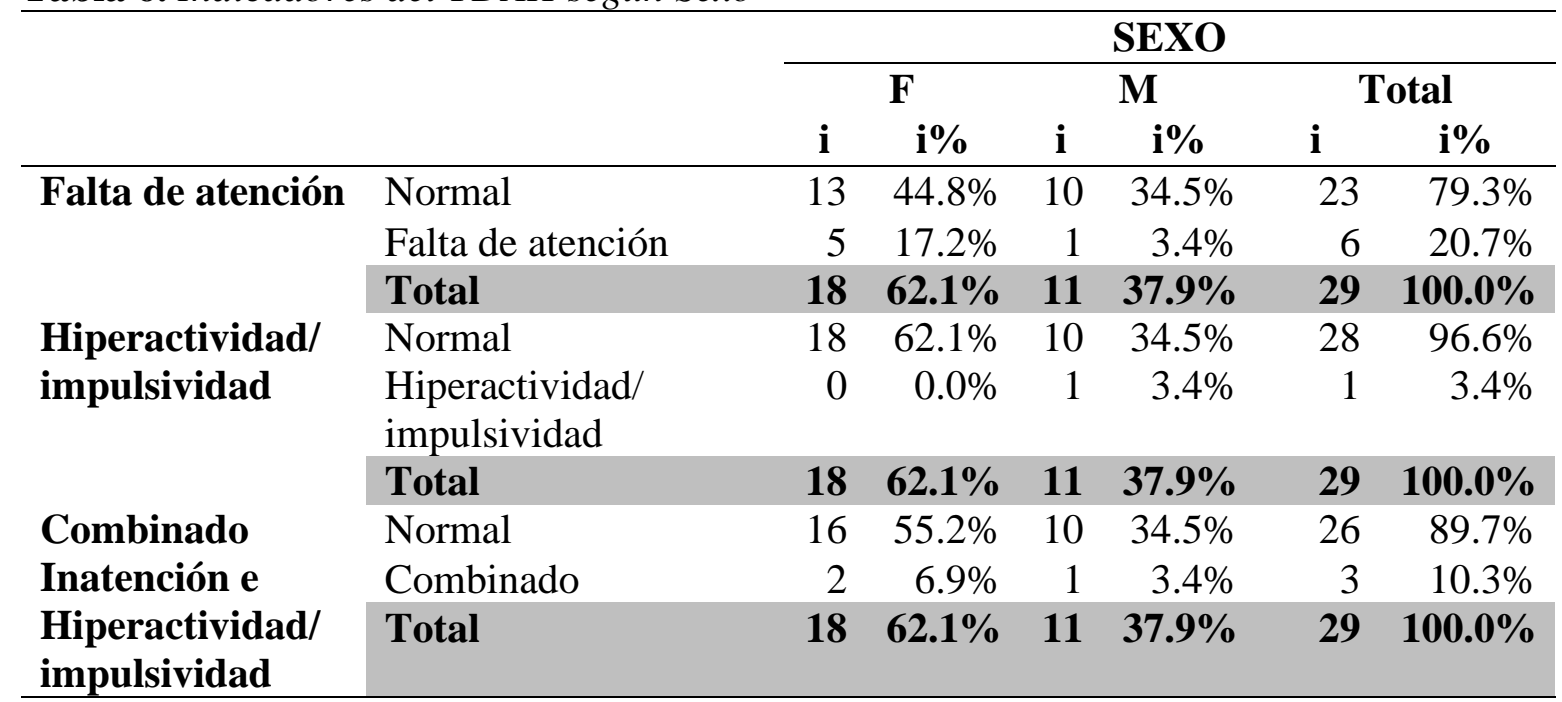

Leyenda: caracterización del déficit de atención por presentaciones

Fuente: elaboración propia

La comprensión lectora de textos andinos que se encuentran contextualizados a la población de estudio, también presenta frecuencias que presentamos para su análisis, considerando que en el tercer grado de educación primaria según el diseño curricular nación de la educación básica del Perú, el estudiante debería de tener un nivel de comprensión lectora esperado con intervalos de calificación entre [14.00 - 17.00] sin embargo podemos observar en la Tabla 7 que el $69.0 \%$ de los niños y niñas en el estudio presentan un nivel de inicio en comprensión lectora, presentándose este nivel con mayor frecuencia en las niñas con un $44.8 \%$, por otro lado en los niños representa el $10.3 \%$ con esta clasificación en el nivel de comprensión lectora.

Tabla 7. Calificación de la Comprensión Lectora de Textos Andinos

\begin{tabular}{|c|c|c|c|c|c|c|}
\hline & \multicolumn{6}{|c|}{ SEXO } \\
\hline & \multicolumn{2}{|r|}{$\mathbf{F}$} & \multicolumn{2}{|c|}{ M } & \multicolumn{2}{|c|}{ Total } \\
\hline & i & i\% & i & i\% & i & i\% \\
\hline Inicio $<=10,00$ & 13 & $44.8 \%$ & 7 & $24.1 \%$ & 20 & $69.0 \%$ \\
\hline Proceso $11,00-13,00$ & 3 & $10.3 \%$ & 3 & $10.3 \%$ & 6 & $20.7 \%$ \\
\hline
\end{tabular}




\begin{tabular}{clrrrrrr}
\hline Nivel de & Esperado $14,00-17,00$ & 2 & $6.9 \%$ & 1 & $3.4 \%$ & 3 & $10.3 \%$ \\
Compresión & Destacado 18,00+ & 0 & $0.0 \%$ & 0 & $0.0 \%$ & 0 & $0.0 \%$ \\
\cline { 2 - 8 } Lectora & Total & $\mathbf{1 8}$ & $\mathbf{6 2 . 1 \%}$ & $\mathbf{1 1}$ & $\mathbf{3 7 . 9 \%}$ & $\mathbf{2 9}$ & $\mathbf{1 0 0 . 0 \%}$ \\
\hline
\end{tabular}

Leyenda: Caracterización de la comprensión lectora de textos andinos

Fuente: elaboración propia

Este estudio considera importante presentar la clasificación de las calificaciones según los desempeños de la comprensión lectora en los niños y niñas según el sexo, identificado que las niñas en el desempeño de obtención de información y el desempeño de reflexión y evaluación tienen un nivel de destacado con un $41.4 \%$ y $27.6 \%$ respectivamente sin embargo en el desempeño de inferencia e interpretación no se presenta ningún caso destacado. Por otro lado, los niños presentan solo en un $27 \%$ de ellos logra el nivel mínimo esperado en el desempeño de obtención de información, y solo un 6.9\% (2 niños) están en el nivel de destacado en el desempeño de reflexión y evaluación por otro lado en el desempeño de inferencia e interpretación el 100\% de los niños no alcanzan ni el nivel mínimo en este desempeño pese a los estándares propuestos por el Ministerio de Educación en el Perú.

Tabla 8. Calificación de la comprensión lectora por desempeños

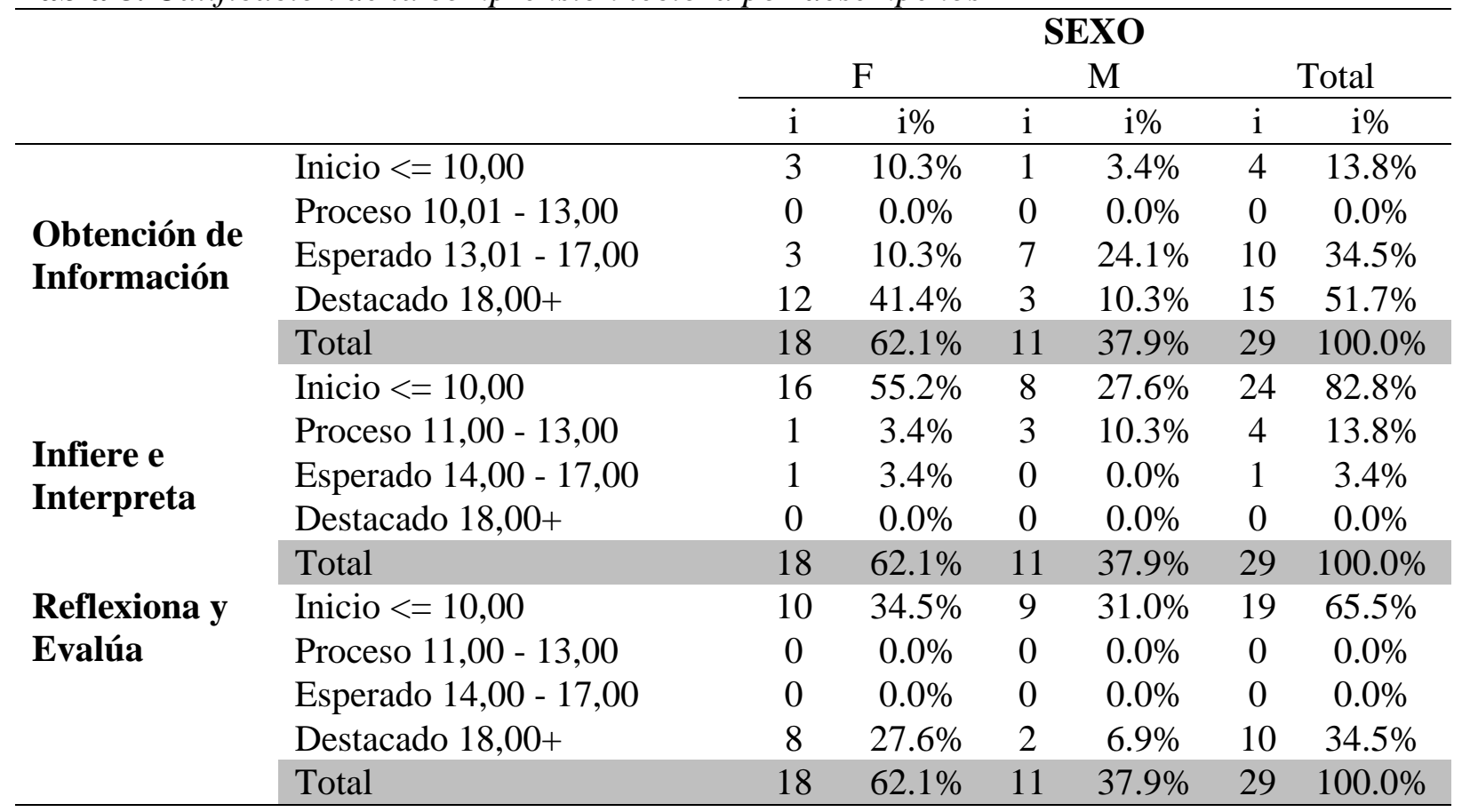

Leyenda: Calificación de la Compresión Lectora por capacidades según los niveles alcanzados en el desarrollo de la competencia lee diversos tipos de textos

Fuente: Elaboración propia

\section{RESULTADOS Y DISCUSIÓN}

El aporte principal de este estudio pretende cambiar la mirada de los docentes a aquellos niños que son tachados o etiquetados como el más travieso, inquieto, desordenado o difíciles. Ahora observamos el problema trastorno de déficit de atención (TDAH) que presentan los niños y niñas y es probable que estas condiciones estén afectando el desarrollo de su aprendizaje en la comprensión lectora. Para ver la frecuencia del déficit de atención y la comprensión de textos andinos, los resultados sugieren que en ambas variables presentan dificultades sobre todo en los 
niños y niñas en los que se deberá analizar los indicadores evaluados de estas variables. Se observó que los niños y niñas al momento de realizar la comprensión de los textos narrativos presentan dificultades para mantener la concentración se movilizan, conversan, se distraen con facilidad y no siguen la comprensión de los cuentos andinos lo cual se refleja en los resultados presentados. Lo que concuerda con los hallazgos de De groot et al. (2017) la discapacidad de lectura (RD) y el trastorno por déficit de atención / hiperactividad (TDAH) son comórbidos y genéticamente correlacionados especialmente la dimensión de falta de atención del TDAH (TDAH-I) para cada trastorno fueron moderadas por la comorbilidad lo que indica no existen genes compartidos. Como también según Christien et al. (2013) que dice que puede existir alguna relación si existe alguna discapacidad de lectura tanto el TDAH como el RD (la discapacidad de lectura) se asociaron con deficiencias en la inhibición y la decisión léxica, Los déficits de memoria de trabajo visoespaciales fueron específicos para niños con TDAH. En cambio a diferencia de Torres (2011) en su investigación no encontró un relación directa entre el déficit de atención con la comprensión lectora solo en algunos aspectos Los niños con TDAH no presentaron rendimientos estadísticamente inferiores al grupo control en el proceso de comprensión lectora, tarea donde en otras investigaciones se ha reportado que al implicar habilidades como identificar letras y palabras, fluidez, vocabulario, y comprender textos, se han evidenciado dificultades que afectan su proceso escolar.

Como también revisando estudios yendo al ámbito de la medicina como este autor Lionel et al. (2011) indica que el genotipo del TDAH tiene un gen de riesgo de heredabilidad en un 34,35 y otra sustento por parte del artículo de Rosenberg et al. (2013)la discapacidad de lectura (RD) y el trastorno por déficit de atención/ hiperactividad (TDAH) no existen genes compartidos por lo que el autor dice que los genes específicos de cada trastorno entran en estas interacciones opuestas, e indica que la heredabilidad de estos dos factores no varía en estas dos dimensiones como es la discapacidad de lectura y el trastorno por déficit de atención/hiperactividad por lo que no se da muy clara esta explicación y lo que recomienda es examinar los genes específicos y factores ambientales más específicos que ayuden a resolver esta paradoja.

Así mismo el trabajo de Torres et al., (2016) afirma que el coeficiente intelectual interfiere en la lectura oral o silenciosa y en algunos de los casos explica el desempeño de los niños y niñas en estas dos variables, en lugar de ser explicadas por la influencia de la memoria de trabajo que tenía relación con las que describían la comprensión lectora, no existió relación entre las variables de la memoria de trabajo y comprensión de lectura en voz alta.

Los lazos que se establecen entre el profesor y estudiantes con dificultades específicas de aprendizajes no son las mejores ya que se hacen más conflictivas, problemáticas, menos cálidas así que el profesor prefiere alejarse de estos estudiantes por lo que no se va a generar aprendizajes significativos en estos estudiantes por lo que se sugiere que el profesor brinde mayor atención a estos niños trabajando con estrategias específicas para estas dificultades y dándole herramientas para su progreso tanto en su dificultad como en el aprendizaje y lograr que sean estudiantes autosuficientes (Prino et al., 2016).

Por tal caso es recomendable que a la edad de 7-8 años y coincidiendo con $2^{\circ}-3^{\circ}$ de educación primaria se realice un diagnóstico recomendando el cribado específico para la detección 
de TDAH y otras dificultades relacionadas (Loro et al., 2015). Adicionando a este procedimiento, el instrumento de compresión de lectura contextualizada para evidenciar mejor los resultados.

Una de las dificultades que se presentaron en este estudio fueron las inclemencias del clima y la falta de consideración de variables sociodemográficas, sin embargo, este estudio es el único en la región que tomó como referencia para la comprensión de lectura un texto andino contextualizado a la región lo que realza el tenor de esta investigación y contribuye al conocimiento de los educadores en Latinoamérica.

\section{CONCLUSIONES}

En síntesis, se realizó la evaluación a los niños el déficit de atención y en comprensión lectora, así como se detectó, que presentan dificultades en la adquisición de una lectura adecuada al mismo tiempo no logran captar las ideas principales del texto, pierden fácilmente la atención lo que viene a ser considerado un trastorno TDAH.

Por lo tanto, podemos afirmar la presencia del TDAH y la carente comprensión de textos andinos en los niños y niñas de tercer grado en la institución I E P. N 70003 Sagrado Corazón de Jesús de la ciudad de Puno, por lo que es importante tomar las previsiones contra este trastorno que afectan en la comprensión lectora, pese a ser esta contextualizada para los niños y niñas en el presente estudio.

\section{REFERENCIAS BIBLIOGRÁFICAS}

Aliaga, L. I. (2012). Comprensión lectora y rendimineto académico en comunicación de alumnos de segundo grado de una institución educativa de Ventanilla. Universidad San Ignacio de Loyola.

Alza, C. (2013). Intervenciones actuales en el trastorno por déficit de atención con/sin hiepractividad (TDAH) en niños y adolescentes. (Vol. 2). Universidad de Chile.

American Psychiatric Association. (2012). ¿Qué es el DSM? ¿Afectará la nueva versión DSM-5 al TDAH? Fundacióncadah.Org, 5-7. https://www.fundacioncadah.org/web/articulo/que-esel-dsm-iv-tr-afectara-la-nueva-version-dsm-5-al-tdah.html

Asociación Peruana de Déficit de Atención. (2002). ¿Qué es el TDAH?. https://www.deficitdeatencionperu.com/que-es-el-tdah/

Balbuena, et al. (2014). Protocolo para la detección y evalaución del alumnado con trastorno por deficit de atencion e hiperactividad en el ámbito educativo. Consejería de educación cultura y deporte. http://feaadah.org/docdow.php?id=581

Centro Nacional de Recursos de CHADD. (2013). El TDAH y el DSM-5. Children And Adults Whith Attention-Deficit Hyperactivity Disorder, 7-11, 14.

Christien, J., Severine, Herbert, R., Raymaekers, R. y, \& Oosterlaan, J. (2013). Especial $58^{\circ}$ Congreso AEPNYA -. 206.

De groot, J., PhD, Bos, V. den, Y, V. der M., \& Minnaert, A. (2017). Rapid Naming and Phonemic Awareness in Children With or Without Reading Disabilities and/or ADHD. Journal of 
Learning Disabilities, 50(2), 168-179. https://doi.org/10.1177/0022219415609186

Espina, A., \& Ortego, A. (2004). Guía Práctica para los Trastornos de Déficit Atencional con / sin Hiperactividad. Janssen-ci

Ferrer, R., Chavez, K. A., Gallardo, C. A., Loredo, G. A. y, \& Meneses, K. A. (2017). Apreciacion diagnostica de profesores del trastorno de deficit atencional con hiperactividad (TDAH) en escolares de enseñanza basica pertenecientes a establecimientos municipalizados de la ciudad de Arica. 8, 15. https://doi.org/10.22199/S07187475.2017.0001.00004

FundacionCADAH.org. (2012). Dificultades académicas de los niños con TDAH a lo largo de la escolarización. https://www.fundacioncadah.org/web/articulo/dificultades-academicas-delos-ninos-con-tdah-a-lo-largo-de-la-escolarizacion-.html

Garcia, M, Prieto, L., Santos, J., Monzon, L., Hernandez, A. y, \& San Feliciana, A. (2008). Trastorno por Déficit de Atención e hiperactividad: un problema actual. Anales de Pediatria, 69, 244.

Herrán, M. et al. (2014). Una revisión narrativa de las escalas de evaluación usadas para el diagnóstico del trastorno por déficit de atención e hiperactividad en niños y adolescentes. Med Wave,

https://www.medwave.cl/link.cgi/Medwave/Revisiones/RevisionTemas/5887

Lionel, A., Crosbie, J., Barbosa, N., Goodale, T., Thiruvahindrapuram, B., Rickaby, J., Gazzellone, M., Carson, A. R., Howe, J. L., Wang, Z., Wei, J., Stewart, A. F. R., Roberts, R., Mcpherson, R., Fiebig, A., Franke, A., Schreiber, S., Zwaigenbaum, L., Fernandez, B. A., ... Scherer, S. W. (2011). Rare Copy Number Variation Discovery and Cross- Disorder Comparisons Identify Risk Genes for ADHD. Science Translational Medicine, 3(95).

Loro, M., García, N., Miernau, I. y, \& Quintero, J. (2015). Evaluation and diagnosis of attention deficit hyperactivity disorder. Medicine (Spain), 11(86), 5153-5156. https://doi.org/10.1016/j.med.2015.09.005

Manual Conners, K. (2013). En qué consisten las Escalas de Conners para evaluar el TDAH. Tdahytu. Torre Europa pasea de la castellana

Martínez, F. J. (2016). Utilidad de los Neuroesteroides en el diagnóstico y/o respuesta a Metilfenidato en pacientes pediátricos con Trastorno por Déficit de Atención con/sin Hiperactividad. Universidad de Granada

Ministerio de Educación del Perú. (2016). Currículo Nacional de la Educación Basica. http://www.minedu.gob.pe/curriculo/pdf/curriculo-nacional-de-la-educacion-basica.pdf

Orjales, I. (1999). Déficit de Atención con Hiperactividad. "Manual para padres y educadores" Madrid - España. CEPE . Madrid . 1-20.

Prino, L. E., Pasta, T., Gastaldi, M., Giovanna, F. y, \& Longobardi, C. (2016). The Effect of Autism Spectrum Disorders, Down Syndrome, Specific Learning Disorders and 
Hyperactivity and Attention Deficits on the Student-Teacher Relationship TT - Incidencia de Trastornos del Espectro Autista, Síndrome Down, Dificultades Específicas d. Revista Electrónica de Investigación Psicoeducativa y Psicopedagógica, 14(38), 89-106. http://dx.doi.org/10.14204/ejrep.38.15043

Rivas, J. A. (2009). Cuentos, Mitos y Relatos. Sagitario

Rosenberg, J., Pennington, B. F., Willcutt, E. G., \& Olson, R. K. (2013). Gene by environment interactions influencing reading disability $(R D)$ and the inattentive symptom dimension of attention deficit / hyperactivity disorder (ADHD). 53(3), 243-251. https://doi.org/10.1111/j.1469-7610.2011.02452.x

Torres, A. M. (2011). Memoria de trabajo y comprension lectora en niños de tercer grado A con trastorno por deficit de atencion/hiperactividad (Issue July).

Torres, A. M., Zuluaga, J. B. y, \& Vilma, V. (2016). Memoria de trabajo y comprension lectora en niños de tercero a quinto grado de primaria con trastorno por déficit atencional / hiperactividad. 12, 23.

Vallés, A. (2005). Comprensión lectora y procesos psicológicos. Comprensión Lectora y Procesos Psicológicos, 49-61.

\section{Conflicto de intereses / Competing interests:}

Los autores declaran que no incurren en conflictos de intereses.

Rol de los autores / Authors Roles:

Grecia Mamani: conceptualización, curación de datos, análisis formal, adquisición de fondos, investigación, metodología, administración del proyecto, recursos, software, supervisión, validación, visualización, escritura preparación del borrador original, escritura - revisar \& amp; edición.

Ana Vilca: conceptualización, análisis formal, investigación, metodología, administración del proyecto, recursos, software, supervisión, validación, visualización, escritura - preparación del borrador original, escritura - revisar \& amp; edición.

Fred Torres: conceptualización, investigación, metodología, administración del proyecto, recursos, software, supervisión, validación, visualización, escritura - preparación del borrador original, escritura - revisar \& amp; edición.

\section{Fuentes de financiamiento / Funding:}

Los autores declaran que no recibieron un fondo específico para esta investigación.

\section{Aspectos éticos / legales; Ethics / legals:}

Los autores declaran no haber incurrido en aspectos antiéticos, ni haber omitido aspectos legales en la realización de la investigación. 